\title{
Primary cilia mediate KIf2-dependant Notch activation in regenerating heart
}

\author{
Xueyu $\mathrm{Li}^{1}$, Qiang $\mathrm{Lu}^{2}$, Yuanyuan Peng ${ }^{1}$, Fang Geng ${ }^{1}$, Xuelian Shao ${ }^{1}$, Huili Zhou ${ }^{2}$, \\ Ying $\mathrm{CaO}^{3}$, Ruilin Zhang ${ }^{4,5 \bowtie}$ \\ ${ }^{1}$ School of Life Sciences, Fudan University, Shanghai 200433, China \\ ${ }^{2}$ Shanghai Medical College, Fudan University, Shanghai, China \\ ${ }^{3}$ Department of Molecular and Cell Biology, School of Life Sciences and Technology, Tongji University, Shanghai 200331, \\ China \\ ${ }^{4}$ School of Basic Medical Sciences, Wuhan University, Wuhan 430072, China \\ ${ }^{5}$ State Key Laboratory of Genetic Engineering, Fudan University, Shanghai 200433, China \\ $凶$ Correspondence: zhangruilin@whu.edu.cn (R. Zhang)
}

Received December 20, 2019 Accepted February 7, 2020

\begin{abstract}
Unlike adult mammalian heart, zebrafish heart has a remarkable capacity to regenerate after injury. Previous study has shown Notch signaling activation in the endocardium is essential for regeneration of the myocardium and this activation is mediated by hemodynamic alteration after injury, however, the molecular mechanism has not been fully explored. In this study we demonstrated that blood flow change could be perceived and transmitted in a primary cilia dependent manner to control the hemodynamic responsive klf2 gene expression and subsequent activation of Notch signaling in the endocardium. First we showed that both homologues of human gene $K L F 2$ in zebrafish, klf2a and $k l f 2 b$, could respond to hemodynamic alteration and both were required for Notch signaling activation and heart regeneration. Further experiments indicated that the upregulation of klf2 gene expression was mediated by endocardial primary cilia. Overall, our findings reveal a novel aspect of mechanical shear stress signal in activating Notch pathway and regulating cardiac regeneration.
\end{abstract}

KEYWORDS heart regeneration, hemodynamics, klf2, Notch signaling, primary cilia

Electronic supplementary material The online version of this article (https://doi.org/10.1007/s13238-020-00695-w) contains supplementary material, which is available to authorized users.

\section{INTRODUCTION}

Adult mammalian heart cannot replenish lost cardiomyocytes (CMs) resulted from myocardial infarction (MI) which could eventually lead to heart failure (Thygesen et al., 2007). By contrast, zebrafish have a strong capacity to regenerate injured heart through dedifferentiation and proliferation of pre-existing CMs (Poss et al., 2002; Jopling et al., 2010; Kikuchi et al., 2010). Notch signal pathway plays a pivotal role in multiple tissue repair processes including zebrafish heart regeneration (Raya et al., 2003). After genetic ablation or surgical resection, Notch signaling is activated in the endocardium of injured heart and subsequently leads to heart regeneration, whereas blockage of such activation impedes this regenerative process (Zhang et al., 2013; Zhao et al., 2014). Despite extensive studies have been carried out on the function of Notch signaling in heart regeneration, the molecular mechanism how it is activated has not been fully elucidated yet (Felician et al., 2014; Nemir et al., 2014; Munch et al., 2017; Zhao et al., 2019).

Hemodynamic force is crucial for the formation of heart valves and cardiac trabeculae, the development and maintenance of hematopoietic stem cells, and the cause of various cardiovascular diseases, frequently acting through Notch signal pathway (Vermot et al., 2009; Nixon et al., 2010; Samsa et al., 2015; Liu et al., 2019). Many factors have been reported to perceive and transmit mechanical shear stress signal, including primary cilia, mechanicalsensitive ion channels, cell adhesion molecules, protein receptor family, transcription factors, etc (Baratchi et al., 2017). Galvez-Santisteban et al. recently reported alteration 
of intracardiac blood flow and hemodynamic shear-stress after ventricle ablation, which was essential for endocardial Notch activation (Gálvez-Santisteban et al., 2019). This activation was mediated by klf2a, zebrafish homologue of the human Krüppel-like factor 2 gene (KLF2) which encodes a well-known hemodynamic responsive transcription factor playing a crucial regulatory role in vascular and cardiac morphogenesis in vivo (Lee et al., 2006; Goddard et al., 2017). However, the function of the other homologue $k$ lf $2 b$ or the identity of factors which perceive and transmit mechanical shear stress signal in this process remains to be elucidated.

This study is aimed to explore the underlying molecular mechanisms of sensation and transmission of mechanical hemodynamic signal to Notch activation during cardiac regeneration. First we demonstrated that both klf2a and klf2 $b$ could respond to hemodynamic alteration, whereas Notch signaling activation and heart regeneration were impeded in klf2 single or double mutants. Further experiments indicated that endocardial primary cilia mediated the upregulation of klf2 gene expression and the subsequent activation of Notch signal pathway. Overall, our findings reveal a novel aspect of mechanical shear stress signal in stimulating Notch activation and regulating cardiac regeneration.

\section{RESULTS}

Reduced blood flow attenuates Notch signaling activation and inhibits ventricle regeneration

To visualize the transient activation of Notch signaling in the endocardium of regenerating hearts, we used a reporter line Tg(tp1:d2GFP) expressing destabilized GFP in Notch-activated cells, to cross with a ventricle ablation line $\mathrm{Tg}$ (vmhc: mCherry-NTR) (Clark et al., 2012; Zhang et al., 2013). DMSO-treated control group displayed strong Notch signals in the atrioventricular canal (AVC) and weak signals in the outflow tract (OFT) (Supplementary figure 1A-C). After ventricle injured with metronidazole (MTZ) treatment at 3 days post fertilization (dpf), Notch signaling was activated in the ablated heart mainly in the AVC and the ventricle, also extending from the AVC to the atrium in various degrees (Supplementary figure 1D-F).

Galvez-Santisteban et al. recently displayed increased level of oscillatory fluctuations of anterograde and retrograde intracardiac flow in injured heart by particle image velocimetry (PIV) analyses (Gálvez-Santisteban et al., 2019) and proved this high oscillatory flow was required for Notch activation. To temporarily inhibit blood flow after ventricle ablation, we utilized Tricaine and 2,3-Butanedione monoxime (BDM), two muscle relaxants affecting electrical function and myosin contraction respectively. Larvae were treated with Tricaine or BDM at 15-24 hpt to achieve most significant effect in ablated group yet with no adverse phenotype observed in non-ablated control group. Notch signal activation was totally blocked in the $24 \mathrm{hpt}$ ablated heart right after Tricaine or BDM treatment (Supplementary figure 1GJ). Whole-mount in situ hybridizations (WISH) also showed significantly attenuated notch $1 b$ mRNA upregulation in ablated hearts at $24 \mathrm{hpt}$ with Tricaine or BDM treatment (Supplementary figure $1 \mathrm{~K}-\mathrm{P}$ ).

This brief inhibition of blood flow and Notch signaling was sufficient to disturb heart regeneration. Compared to ablated larvae without treatment, the ventricular fluorescence and heart morphology of ablated larvae treated with Tricaine or BDM could not restore at 4 days post treatment (dpt) (Supplementary figure 2). Quantification of regeneration rate at 4 dpt showed that the percentage of recovered larvae after reducing blood flow dropped to $20 \%$ in Tricaine treated ablated group $(\mathrm{N}=336)$ and $21 \%$ in BDM treated ablated group ( $N=126)$, significantly lower than the $83 \%$ recovery rate in wildtype ablated group ( $N=249$ ) (Supplementary figure $1 Q$ ). Overall, our results confirmed that hemodynamic force was essential for the activation of Notch signaling and ventricle regeneration in agreement with recent finding by Galvez-Santisteban et al. (Gálvez-Santisteban et al., 2019).

\section{Reduced blood flow changes $k l f 2 a$ and $k l f 2 b$ gene expression during ventricle regeneration}

KLF2 is well-known for its responsiveness to hemodynamic alteration during mammalian development and various disease progressions (Dekker et al., 2002). The zebrafish homologue klf2a has been proved to play a conserved role in similar settings (Steed et al., 2016), however, the function of the other homologue $k / f 2 b$ has not been fully characterized (Oates et al., 2001). We first used WISH to compare the expression profiles of both homologues during ventricle regeneration. Both $k l f 2 a$ and $k l f 2 b$ genes were expressed weakly in the AVC and OFT of control larvae hearts at $4 \mathrm{dpf}$, then klf2a expression decreased from 5 to $7 \mathrm{dpf}$ whereas $k l f 2 b$ expression remained constant (Fig. 1A-D, I-L). After cardiac injury at $1 \mathrm{dpt}$, klf2a expression was significantly upregulated in the AVC where the level of oscillatory flow increased the most. From 2 to $4 \mathrm{dpt}$, klf2a expression was gradually weakened in the AVC, but the range spread to the ventricle and atrium (Fig. 1E-H). By contrast, the expression of $k$ lf $2 b$ in ablated hearts was slightly upregulated in the AVC at $1 \mathrm{dpt}$, which maintained a constant level and did not extend to either chamber at later stages (Fig. 1M-P). We then examined how klf2a and $k$ lf $2 b$ genes responded to reduced blood flow. The upregulation of klf2a mRNA in the AVC of ablated hearts at $1 \mathrm{dpt}$ was attenuated upon Tricaine or BDM treatment (Fig. 1Q-V). Interestingly, klf2 $b$ expression in some of the Tricaine or BDM treated control and ablated hearts was slightly enhanced and expanded from the AVC (Fig. 1W-B'). These results suggested that klf2a and $k l f 2 b$ genes responded differently to blood flow alteration and might play different roles during cardiac regeneration. 

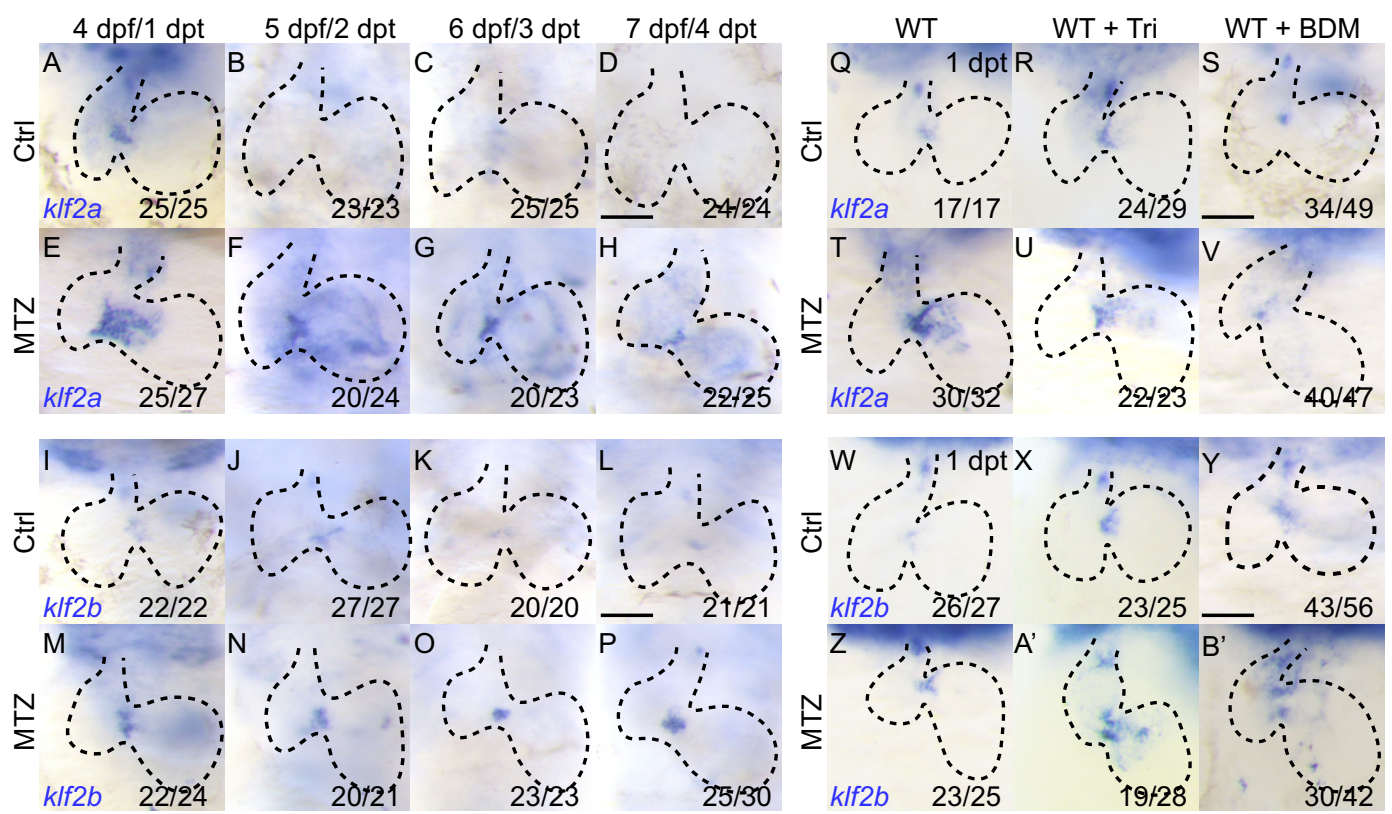

Figure 1. Reduced blood flow changes $k l f 2 a$ and $k$ lf2 $b$ expression during ventricle regeneration. (A-H) Whole-mount in situ hybridizations showing klf2a upregulation in ablated hearts $(E-H)$ compared to control hearts (A-D) at 4-7 dpf/1-4 dpt. (I-P) Wholemount in situ hybridizations showing klf2b upregulation in ablated hearts (M-P) compared to control hearts (I-L) at 4-7 dpf/1-4 dpt. $(Q-V)$ Whole-mount in situ hybridizations indicated klf2a upregulation after ablation $(Q, T)$ was blocked in Tricaine treated hearts $(R$, $\mathrm{U}$ ) and BDM treated hearts (S, V) at $1 \mathrm{dpt}$. (W-B') Whole-mount in situ hybridizations indicated klf2b upregulation after ablation (W, Z) was slightly enhanced in Tricaine treated hearts $\left(X, A^{\prime}\right)$ and BDM treated hearts (Y, B') at $1 \mathrm{dpt}$. Scale bars, $50 \mu \mathrm{m}$. dpt, days post treatment. Dashed lines outline the hearts. Numbers indicate the ratio of representative staining observed.

\section{klf2 mutants result in compensation of homologue expression}

To further investigate the role of $k l f 2 a$ and $k l f 2 b$ in the regulation of cardiac regeneration, we generated mutants using CRISPR/Cas9 genome editing technique. We first obtained $\mathrm{klf2a}^{-/-}$frameshift mutants bearing $1 \mathrm{bp}$ deletion which resulted in premature translation termination. The predicted truncated protein consisted of 38 amino acids compared to the full length of 380 amino acids in wildtype Klf2a (Fig. 2A, C). We next obtained $\mathrm{klf}_{2} \mathrm{~b}^{-/-}$mutants bearing 5 bp deletion which produced truncated proteins of 261 amino acids. The $k l f 2 a^{-l-} / k l f 2 b^{-1-}$ double mutants (referred as $k l f 2^{-/-}$double mutants later on) with 1-bp-deletion in klf2a and 7-bp-deletion in $k l f 2 b$ produced truncated proteins of similar size, missing the conserved zinc finger domains (Fig. 2B, D). During the first week of development most larvae of $\mathrm{klf2a}^{-{ }^{-}}$ mutants, $k l f 2 b^{-/-}$mutants and $k l f 2^{-1-}$ double mutants did not show gross morphological defects. We found a small percentage of larvae with cardiac edema in klf2 single and double mutants but no obvious CM extrusion was observed in double mutants, which was different from a previous report (Rasouli et al., 2018). Although $\mathrm{klf2a}^{-/-}$and $\mathrm{klf2}^{-/-}$single mutants could grow to adulthood normally, most of klf2 $^{-1-}$ double mutant fish would die during the second week of development due to unidentified reasons, and only a few could reach sexual maturity but still were prone to death.
To assess the knockout efficiency we performed real time PCR using cDNA from whole mutant larvae at $4 \mathrm{dpf}$ (Fig. 2M, $\mathrm{V}, 4$ independent experiments). klf2a mRNA level was reduced to $0.57 \pm 0.06$ fold of wildtype in $\mathrm{klf}^{2} \mathrm{a}^{-/-}$larvae whereas klf2 $b$ mRNA level had a larger reduction to $0.26 \pm$ 0.05 fold of wildtype in $\mathrm{klf}_{2} \mathrm{~b}^{-/-}$larvae. Interestingly, klf2a mRNA level significantly increased to $2.23 \pm 0.25$ fold in $k l f 2 b^{-1-}$ larvae while $k l f 2 b$ mRNA level showed a similar trend in $k l f 2 a^{-1-}$ larvae with $1.44 \pm 0.30$ fold compared to wildtype, suggesting a possible compensation of klf2 homologue expression in single mutants. The expression level of klf2a or $k l f 2 b$ in $k l f 2^{-/-}$double mutants was between the levels in two single mutants, maybe due to the average of reduction and compensation effects. WISH of $k$ If2a and klf2b mRNA in the hearts of wildtype, klf2a $a^{-1-}$ mutants, $k l f 2 b^{-1-}$ mutants and $\mathrm{klf2}^{-/-}$double mutants at $4 \mathrm{dpf}$ showed a pattern consistent with real time $\mathrm{PCR}$ result (Fig. 2E-H, N-Q).

klf2 mutants result in blunted Notch activation and reduced ventricle regeneration

Next we performed ventricle ablation in all three klf2 homozygous mutants with $T g(v m h c: m C h e r r y-N T R)$ background. Although klf2a was strongly upregulated in $k l f 2 b^{-1-}$ ablated hearts at $24 \mathrm{hpt}$ and vice versa (Fig. $2 \mathrm{I}-\mathrm{L}, \mathrm{R}-\mathrm{U}$ ), the ventricular fluorescence and heart morphology of ablated 
klf2a

klf2a $\overline{\text { CAGAGGTGGAAGGATGAACTGGACAGGTCC }}$ klf2aD1 CAGAGGTGGAAGGATGAACTG - ACAGGTCC

B

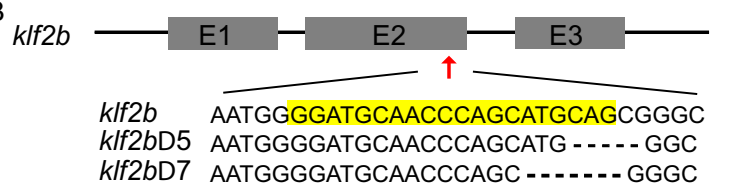

D
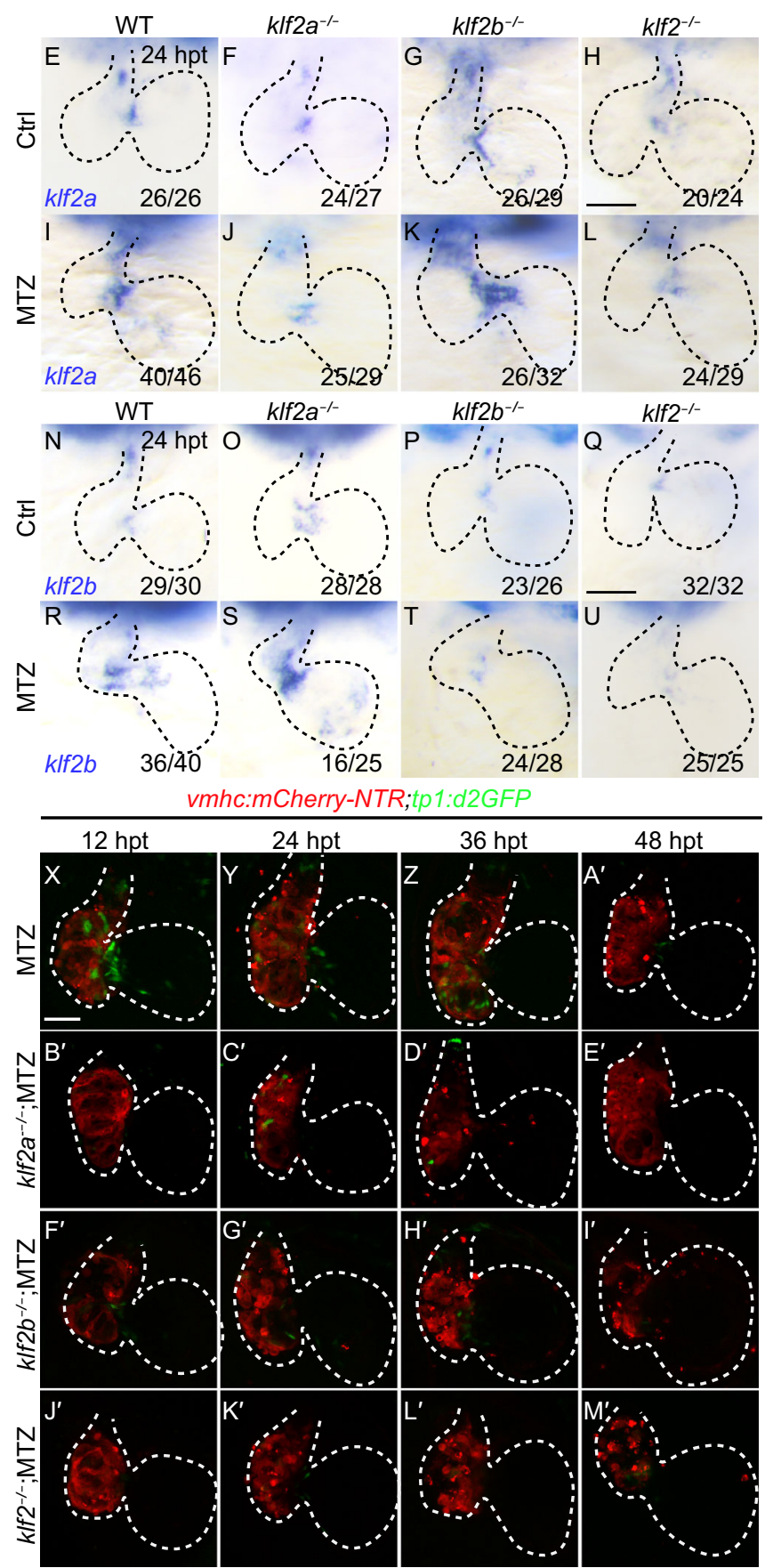

KIf2a

Klf2aD1

Klf2b

KIf2bD5

Transactivation Transrepression

110

Transactivation Transrepressior
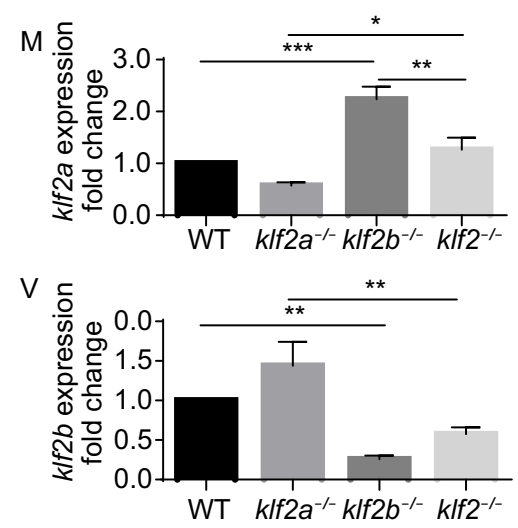

W
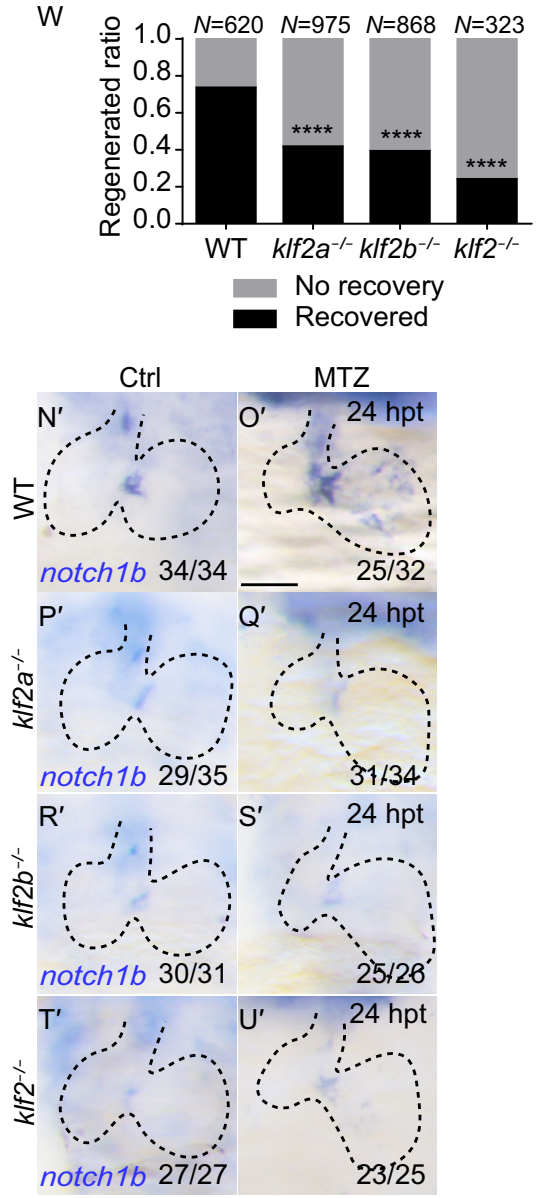
4 Figure 2. klf2 mutants result in compensation of homologue expression, blunted Notch signaling activation and reduced ventricle regeneration. ( $A$ and $B$ ) Schematic diagrams of the zebrafish $k l f 2 a$ and $k l f 2 b$ loci with the sgRNA target site sequence (red arrowheads). (C, D) Functional domain diagrams of wildtype Klf2a or Klf2b and predicted truncated protein in corresponding mutants. (E-L) Whole-mount in situ hybridizations showing klf2a expression pattern in the control and ablated hearts of wildtype, klf2a $a^{-/-}$mutants, klf $2 b^{-/-}$ mutants and klf2 ${ }^{-/-}$double mutants at 24 hpt. (M) Quantification of the fold change of klf2a expression in wildtype and klf2 mutant larvae at $4 \mathrm{dpf}$ by real time PCR. 4 independent experiments. Mean + s.e.m. ANOVA analysis, ${ }^{*} P<0.05$, ${ }^{\star *} P<$ $0.01,{ }^{* *} P<0.001$. (N-U) Whole-mount in situ hybridizations showing klf2 $b$ expression pattern in the control and ablated hearts of wildtype, $k l f 2 a^{-/-}$mutants, $k l f 2 b^{-/-}$mutants and $k l f 2^{-/-}$ double mutants at $24 \mathrm{hpt}$ (V) Quantification of the fold change of $k l f 2 b$ expression in wildtype and klf2 mutant larvae at $4 \mathrm{dpf}$ by real time PCR. 4 independent experiments. Mean + s.e.m. ANOVA analysis, ${ }^{* *} P<0.01$. (W) Quantification of the heart recovery rate (black bars) in ablated wildtype and klf2 mutants at $4 \mathrm{dpt}$. The number of larvae analyzed for each condition is indicated. Binomial test (versus WT), ${ }^{* \star * *} P<0.0001$. (X-M') Confocal stack projections of ablated $T g$ (vmhc:mCherry-NTR; tp1:d2GFP) hearts showing Notch signaling pattern in wildtype $\left(\mathrm{X}-\mathrm{A}^{\prime}\right), k l f 2 a^{-/-}$mutants $\left(\mathrm{B}^{\prime}-\mathrm{E}^{\prime}\right), k l f 2 b^{-/-}$mutants $\left(\mathrm{F}^{\prime}-\mathrm{I}^{\prime}\right)$ and $k^{\prime} 2^{-1-}$ double mutants (J'-M') at 12, 24, 36, 48 hpt. (N'-U') Whole-mount in situ hybridizations indicated notch $1 b$ upregulation as in ablated wildtype hearts ( $\left.\mathrm{N}^{\prime}, \mathrm{O}^{\prime}\right)$ was blocked in $k l f 2 a^{-1-}$ mutant hearts ( $\left.\mathrm{P}^{\prime}, \mathrm{Q}^{\prime}\right), k l f 2 b^{-1-}$ mutant hearts (R', $\left.\mathrm{S}^{\prime}\right)$ and $k l f 2^{-1-}$ double mutant hearts ( $\left.T^{\prime}, U^{\prime}\right)$ at $24 \mathrm{hpt}$. Scale bars, 50 $\mu \mathrm{m}$. hpt, hours post treatment. Dashed lines outline the hearts. Numbers indicate the ratio of representative staining observed.

hearts were not recovered in all three mutants at $4 \mathrm{dpt}$ after injury compared to wildtype (Supplementary figure 2). We quantified the heart regeneration rate at $4 \mathrm{dpt}$ and found the percentage of recovered larvae reduced from $73 \%$ in wildtype ( $\mathrm{N}=620)$ to $42 \%$ in $\mathrm{klf2} \mathrm{a}^{-/-}$mutants $(\mathrm{N}=975)$ and $39 \%$ in $k l f 2 b^{-1-}$ mutants $(\mathrm{N}=868)$. The percentage further reduced to $24 \%$ in $k l f 2^{-/-}$double mutants $(N=323)$ (Fig. 2W).

To examine whether $k / f 2 a$ and $k l f 2 b$ regulated ventricle regeneration through mediating Notch signaling activation, we bred the klf2 mutants to homozygosity in $T g$ (vmhc: mCherry-NTR; tp1:d2GFP) background. In contrast to the strong activation of Notch signaling in the AVC of wildtype hearts after ventricle ablation, no such activation was observed in the three klf2 mutants from 12 to $48 \mathrm{hpt}$ (Fig. 2X-M'). WISH of notch1b expression also exhibited a similar pattern. notch1b expression was significantly upregulated in the AVC and expanded to chambers in wildtype ablated hearts. However, in both control and ablated klf2 mutant hearts, notch $1 b$ was only expressed weakly in the AVC and OFT (Fig. 2N'-U'). These results indicated that both homologues of in human gene KLF2 in zebrafish, klf2a and $k l f 2 b$, were important molecules necessary for Notch signaling activation and cardiac regeneration.

\section{Endocardial primary cilia exist in the hearts at later stages}

Knowing that $k$ lf2a and $k$ lf $2 b$ could respond to hemodynamic alteration and regulate Notch signaling activation as well as ventricle regeneration, we then aimed to explore how blood flow change mediated klf2 gene expression. Primary cilia, among others, are the popular candidate mechanical sensors for shear stress in endothelial cells in various models (Nauli et al., 2008). Previous study revealed the existence of primary cilia in zebrafish heart at $1 \mathrm{dpf}$ (Samsa et al., 2015). However, whether primary cilia still exist at later stages when we perform ventricle ablation study is still unclear. Immunofluorescent staining of acetylated-alpha tubulin, a primary cilia marker, was performed at $3 \mathrm{dpf}$. Multiple signals could be detected at this stage, most in the OFT and AVC region as well as in the ventricle and atrium at lower abundance (Fig. 3A, D). To verify the location of these primary cilia, we performed acetylated-alpha tubulin immunostaining using Tg(flk:GFP), Tg(tcf21:nucGFP) and Tg(vmhc: $m$ Cherry-NTR) fishlines. The results indicated cilia existed in all three layers of the heart, including endocardial cells around AVC (Fig. 3H, K), epicardial cells in OFT (Fig. 3I, L) and ventricular CMs (Fig. 3J, M). We also examined the localization of Arl13b, a small GTPase of the Arf/Arl family that could bind to primary cilia, with immunostaining and reporter line Tg(Ubi:Arl13b-GFP) (Austin-Tse et al., 2013). Although most Arl13b signal was on the plasma membrane, primary cilia-like structure could be detected in the AVC region of $3 \mathrm{dpf}$ zebrafish heart (Supplementary figure 3 ). Thus our results confirmed that cardiac primary cilia existed before ventricle ablation and there was some cilia located in the AVC endocardium where Notch signaling was activated.

We then examined the primary cilia formation in the klf2 mutants with acetylated-alpha tubulin immunostaining (Fig. 3B, C, E-G). The result indicated the number of cilia had no significant change in the hearts of $k / f 2 a^{-/-}$mutants $(166.0 \pm 11.7$ per heart, $\mathrm{N}=11), \mathrm{klf}^{2} \mathrm{~b}^{-/-}$mutants $(144.5 \pm$ 16.5 per heart, $\mathrm{N}=6$ ) and $\mathrm{klf2}^{-/-}$double mutants $(152.4 \pm$ 11.2 per heart, $N=7$ ) compared to wildtype (184.2 \pm 10.7 per heart, $N=16$ ) at $3 \mathrm{dpf}$. We further characterized the primary cilia pattern in the injured Tg(vmhc:mCherry-NTR) larvae hearts (Fig. $3 \mathrm{~N}-\mathrm{V}$ ). The cardiac cilia number in the control group at $4 \mathrm{dpf}$ was dramatically reduced $(85.5 \pm 9.5$ per heart, $\mathrm{N}=11$ ) compared to that of $3 \mathrm{dpf}$ mentioned above (Fig. 3G, V). However, the staining in ablated hearts at $4 \mathrm{dpf} /$ $1 \mathrm{dpt}$ revealed an increase in the cilia number $(123.7 \pm 7.0$ per heart, $\mathrm{N}=11$ ) which could be obliterated by blood flow reduction induced by post-ablation Tricaine treatment $(76.9 \pm$ 6.3 per heart, $N=13$ ). Our results suggested a dynamic pattern of primary cilia formation in the heart during 


\section{Ac-Tubulin flk:GFP}
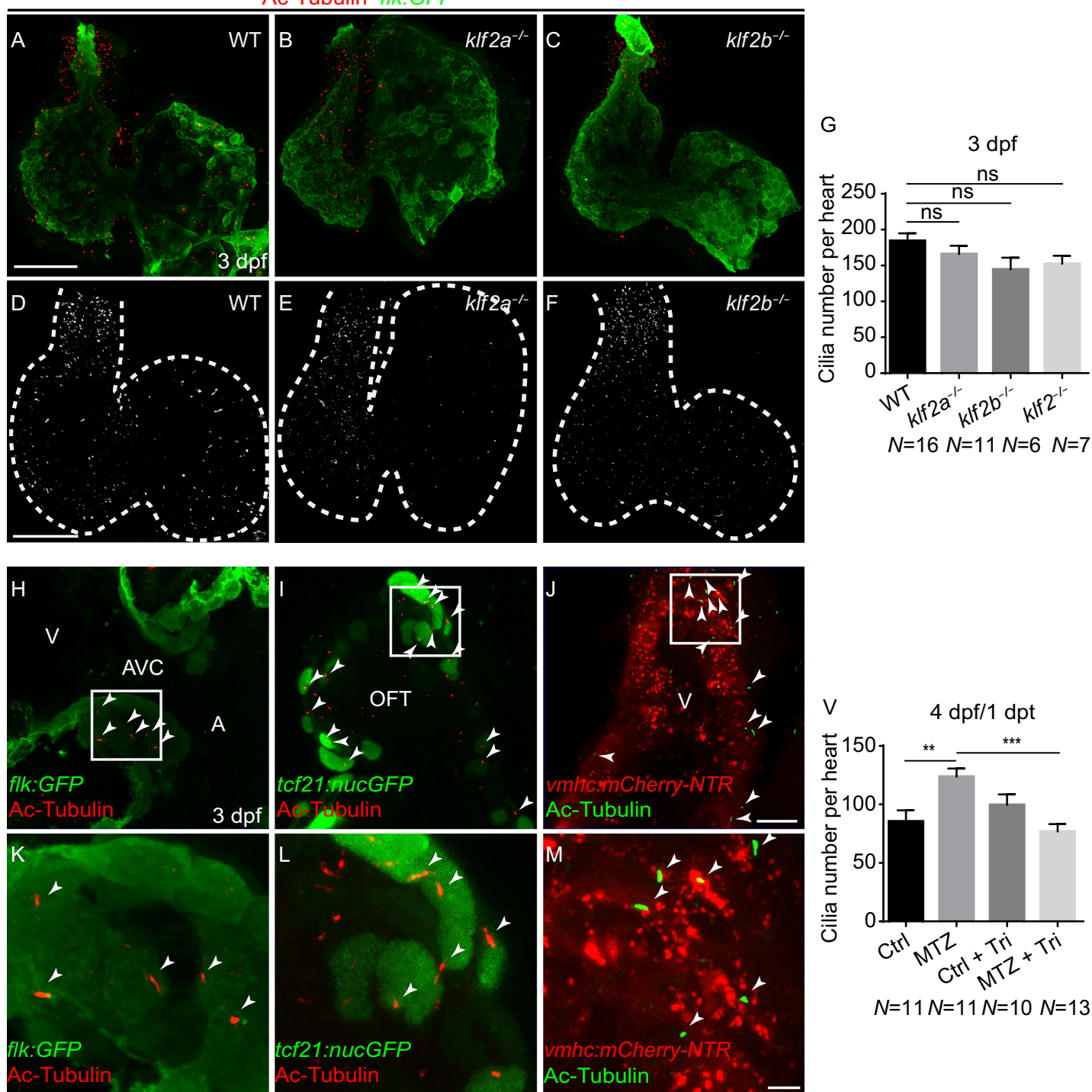

Ac-Tubulin vmhc:mCherry-NTR

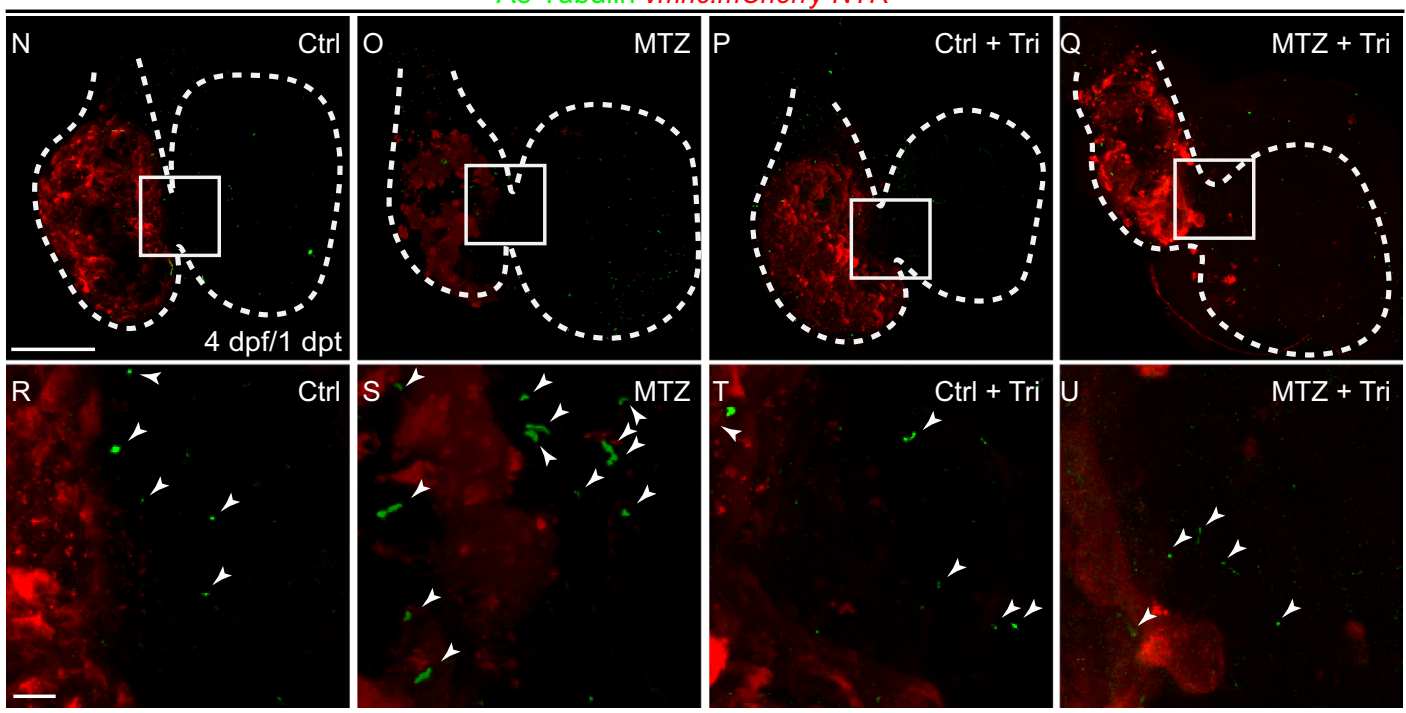


Figure 3. Endocardial primary cilia exist in the hearts at later stages. (A-F) Confocal stack projections of immunofluorescence showing cardiac cilia in wildtype and klf2 mutants at 3 dpf. Green, anti-GFP immunostaining; red, acetylated tubulin immunostaining. (D-F) greyscale of red channel only. (G) Quantification of cardiac cilia number in wildtype and klf2 mutants at $3 \mathrm{dpf}(\mathrm{N}=16,11,6,7$ respectively). Mean + s.e.m. ANOVA analysis, ns, not significant. (H-M) Acetylated tubulin immunostaining showing primary cilia in endocardial cells $(H, K)$, epicardial cells $(\mathrm{I}, \mathrm{L})$, and cardiomyocytes $(\mathrm{J}, \mathrm{M})$ at $3 \mathrm{dpf}$. (K$\mathrm{M})$ magnified white boxes in $(\mathrm{H}-\mathrm{J})$. ( $\mathrm{N}-\mathrm{U})$ Confocal stack projections of immunofluorescence showing cardiac cilia in controlTricaine or BDM treatment or ablated Tg(vmhc:mCherryNTR) hearts with or without Tricaine treatment at $1 \mathrm{dpt}$ ( $(\mathrm{R}-\mathrm{U})$ magnified white boxes in (N-Q). Red, mCherry fluorescence; green, acetylated tubulin immunostaining. (V) Quantification of cardiac cilia number in control or ablated hearts with or without Tricaine treatment at $1 \mathrm{dpt} / 4 \mathrm{dpf}(\mathrm{N}=11,11,10,13$ respectively). Mean + s.e.m. ANOVA analysis, ${ }^{\star \star} P<0.01$, ${ }^{* * *} P<0.001$. Scale bars, $(\mathrm{A}-\mathrm{F}, \mathrm{N}-\mathrm{Q}) 50 \mu \mathrm{m},(\mathrm{H}-\mathrm{J}) 10 \mu \mathrm{m},(\mathrm{K}-$ $\mathrm{M}, \mathrm{R}-\mathrm{U}) 5 \mu \mathrm{m}$. dpf, days post fertilization, dpt, days post treatment. Dashed lines outline the hearts. Arrowheads point to cilia. A, atrium; AVC, atrioventricular canal; OFT, outflow tract; $\mathrm{V}$, ventricle.

development and regeneration, which was probably regulated by hemodynamic alteration.

\section{Cilia knockdown inhibits klf2 and Notch activation during ventricle regeneration}

To further investigate the role of primary cilia in ventricle regeneration, we knocked down the expression of ift88, an important intraflagellar transporter involved in the development and maintenance of primary cilia (Pazour et al., 2000). After ift88 morpholino injection at one cell stage, acetylatedalpha tubulin immunostaining showed the cilia formation was disrupted in the developing inner ear and pronephric duct, two well-known ciliated organs (Supplementary figure 4A, D, $\mathrm{E}, \mathrm{H})$. These phenotypes were similar as seen in ift20 $\mathrm{O}^{-1-}$ and ift172 ${ }^{-1-}$ mutants (Supplementary figure 4B, C, F, G) and assured the effectiveness and specificity of ift88 knockdown. We then examined the primary cilia formation in the hearts of ift $20^{-1-}$, ift $172^{-/-}$mutants and ift88 morphants at $3 \mathrm{dpf}$. The number of cilia was dramatically reduced in ift20 $0^{-1-}$, ift172 ${ }^{-1-}$ mutants and ift88 morphants $(57.4 \pm 8.9,49.9 \pm 5.4,71.9 \pm$ 6.0 per heart, $\mathrm{N}=7,7,10$, respectively) compared to control $(173.1 \pm 5.3$ per heart, $N=15)$ (Figs. 4A-C, Supplementary figure $4 \mathrm{I}-\mathrm{M})$. These results confirmed that ift88 knockdown disrupted cardiac primary cilia formation as in other ift family gene mutants.

We further performed ventricle ablation in ift88 $\mathrm{MO}$ injected Tg(vmhc:mCherry-NTR) larvae to examine the activation of klf2 expression and Notch signaling. Although klf2a expression was upregulated in the ventricle of ift88 morphants without injury, the activation of klf2a and $k l f 2 b$ as seen in the ablated hearts of control morphants was inhibited in the ablated hearts of ift88 morphants (Fig. 4D-K). Similarly, Notch signaling activation in injured hearts was also blocked in ift88 morphants, revealed by WISH of notch $1 \mathrm{~b}$ mRNA and $\operatorname{Tg}(t p 1: d 2 G F P)$ reporter line (Fig. 4L-S). To examine the effect of cilia knockdown on the expression of cardiac transcription factors after heart injury, we performed WISH of hand 2 and $n k x 2.5$. The results showed both genes were highly enhanced in the ablated hearts of control morphants, whereas these activation were blunted in the ablated hearts of ift88 morphants (Supplementary figure 5). Taken together, these findings confirmed the requirement of cardiac primary cilia for the activation of klf2 and Notch signaling during ventricle regeneration.

\section{DISCUSSION}

In this study we have revealed the unique role of mechanosensation pathway in regulating cardiac regeneration. After ventricle ablation, hemodynamic alteration is perceived by endocardial cells through primary cilia, which mediates the upregulation of hemodynamic responsive factors $k l f 2 a$ and $k l f 2 b$. The increased klf2 gene expression in turn activates endocardial Notch signaling to promote ventricle regeneration (Fig. 5, left panel). When blood flow is significantly reduced by Tricaine or BDM treatment, or disruption of cardiac primary cilia formation occurs as in ift88 morphants, there is no sufficient extrinsic mechanical signal to be sensed or transmitted to stimulate klf2 upregulation which results in impairment of Notch signaling activation and failure of heart regeneration (Fig. 5, middle panel). If the klf2 genes are knocked out, Notch signaling activation is also blocked and the heart will not be able to regenerate normally (Fig. 5, right panel).

\section{$k l f 2 a$ and $k$ lf $2 b$ both respond to hemodynamic alteration}

The KLF proteins of Krüppel-like factor family are widely expressed transcriptional regulators which play important roles in hematopoiesis, angiogenesis, fin and epidermal development (Atkins and Jain, 2007). Specifically, KLF2 is one of the most popular blood flow responsive molecules (Dekker et al., 2002). Goddard et al. have revealed that hemodynamic forces affect heart valve development through the KLF2-WNT9B paracrine signaling axis in mice, and klf2a, one of the two KIf2 homologue genes in zebrafish, performs a similar function (Goddard et al., 2017). klf2a can also regulate fibronectin synthesis by participating in mechanical conduction to affect zebrafish heart valve morphogenesis (Steed et al., 2016). However, klf2b, the other zebrafish homologue, obtains much less attention. Recently Rasouli et al. have reported that while single mutants of $k l f 2 a^{-1-}$ or $k l f 2 b^{-/-}$exhibit no gross defects, $k l f 2^{-/-}$double mutants display a novel phenotype of $\mathrm{CM}$ extrusion towards the abluminal side which also requires cardiac contractility 

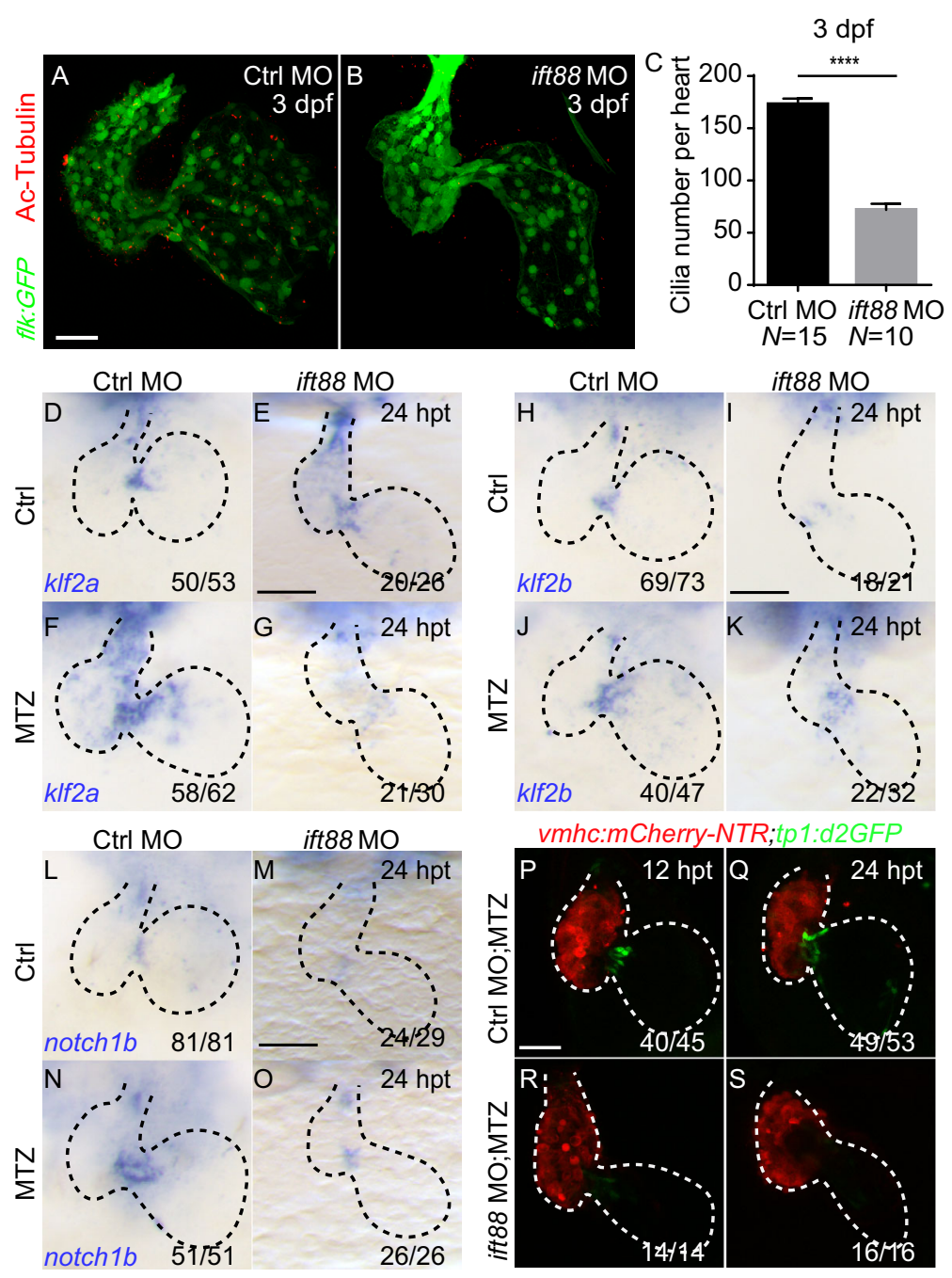

Figure 4. Cilia knockdown inhibits Notch signaling and klf2 activation during ventricle regeneration. (A, B) Acetylated tubulin immunostaining in $T g(f l k: G F P)$ heart showing cardiac cilia in control morphants (A) and ift88 morphants (B) at 3 dpf. Green, anti-GFP immunostaining; red, acetylated tubulin immunostaining. dpf, days post fertilization. (C) Quantification of cardiac cilia number in control morphants and ift88 morphants at $3 \mathrm{dpf}\left(\mathrm{N}=15,10\right.$ respectively). Mean + s.e.m. Student's $t$-test, ${ }^{* * * *} P<0.0001$. (D-G) Whole-mount in situ hybridizations indicated klf2a upregulation in control morphant ablated hearts (F) compared to control hearts (D) at $24 \mathrm{hpt}$, whereas this activation was blocked in ablated ift88 morphant hearts (G). (H-K) Whole-mount in situ hybridizations indicated $k l f 2 b$ upregulation in control morphant ablated hearts $(\mathrm{J})$ compared to control hearts $(\mathrm{H})$ at $24 \mathrm{hpt}$, whereas this activation was blocked in ablated ift88 morphant hearts $(\mathrm{K})$. (L-O) Whole-mount in situ hybridizations indicated notch1b upregulation in control morphant ablated hearts $(\mathrm{N})$ compared to control hearts $(\mathrm{L})$ at $24 \mathrm{hpt}$, whereas this activation was blocked in ablated ift88 morphant hearts (O). (P-S) Confocal stack projections of ablated Tg(vmhc:mCherry-NTR; tp1:d2GFP) hearts indicated Notch signaling activation was inhibited in ift88 morphants at 12-24 hpt. Scale bars, $50 \mu \mathrm{m}$. hpt, hours post treatment. Dashed lines outline the hearts.

Numbers indicate the ratio of representative staining observed.

(Rasouli et al., 2018). However, we did not notice obvious $\mathrm{CM}$ extrusion in our double mutants, presumably due to various truncated products in different mutant alleles. Most of our $\mathrm{klf2}^{-/-}$double mutant larvae cannot survive into adulthood which is consistent with previous report (Rasouli et al., 2018). In our study we have shown both klf2a and klf2b can respond to hemodynamic alteration after ventricle injury. Expression of both genes initially increases in the AVC region while only klf2a expands to the ventricle and atrium at later stages. Interestingly the expression of klf2a in $k l f 2 b^{-l-}$ mutants or klf2b in $k l f 2 a^{-1-}$ mutants is upregulated during heart regeneration, probably due to compensation for the loss of the other paralogue. However, both klf2a and $k$ lf $2 b$ are required for endocardial Notch activation since such activation is blocked in single mutants which results in regenerative failure. We speculate that KIf2a and Klf2b may 


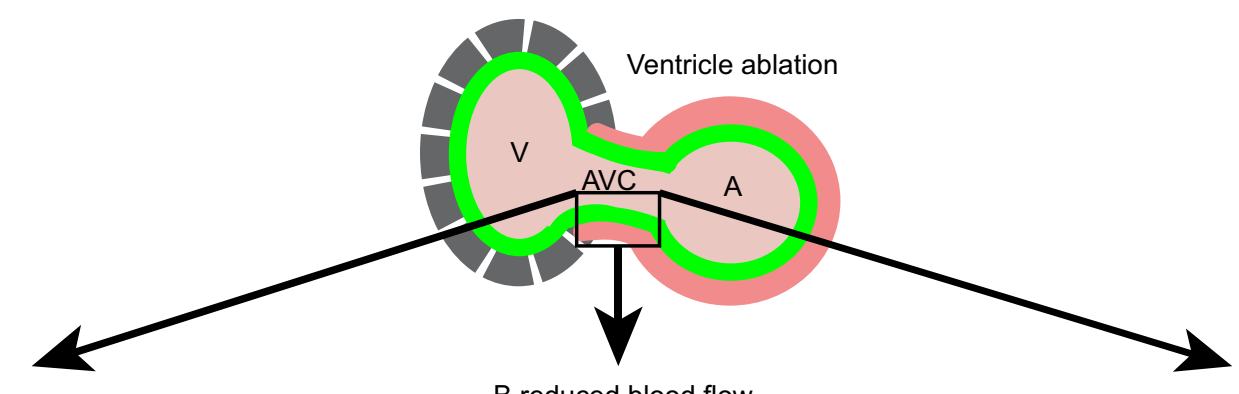

A wildtype
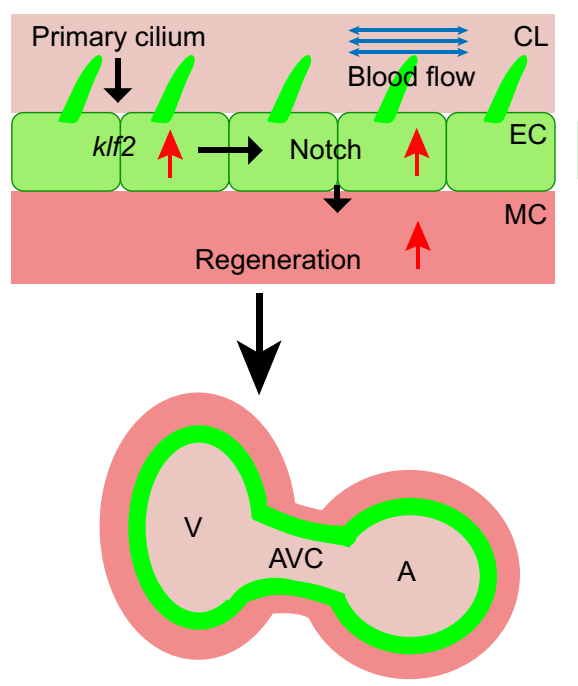

Ventricle regeneration success
$B$ reduced blood flow or cilia KD
C klf2 mutant

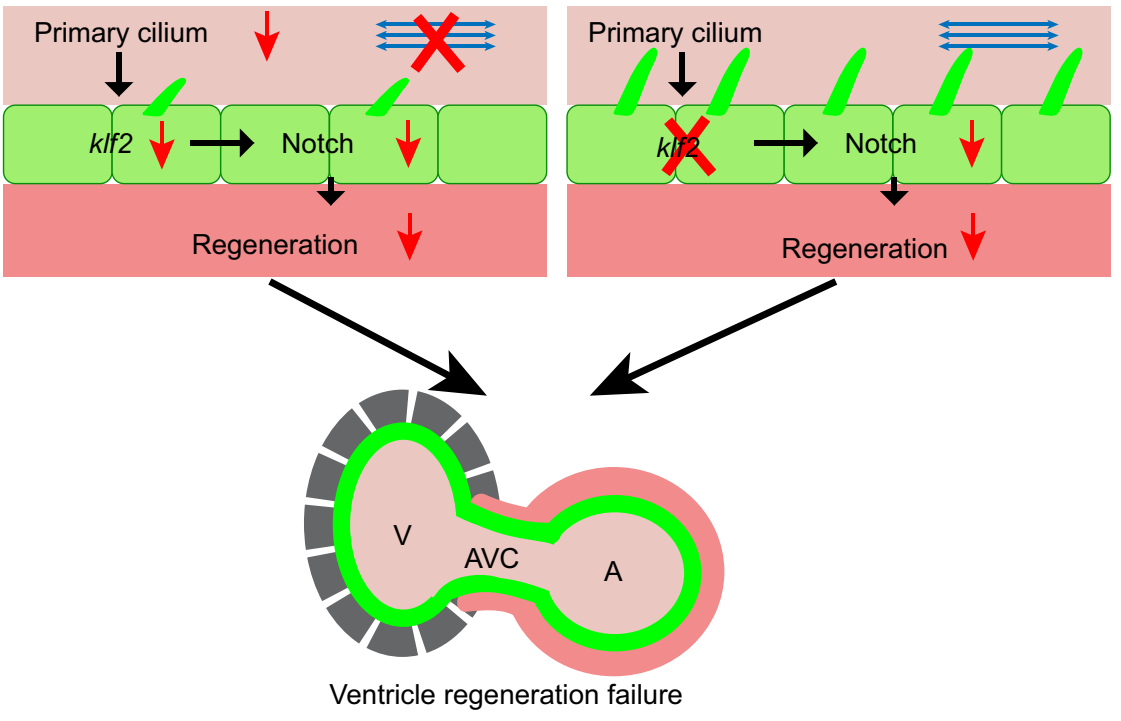

Figure 5. Diagrams of hemodynamic-responsive Klf2-dependant Notch activation in ventricle regeneration. (A) During normal ventricle regeneration process, primary cilia on the endocardial cells (green) sense the oscillatory blood flow (blue arrows), which leads to upregulation of the klf2 and subsequent activation of Notch signaling in the endocardium. This Notch activation is essential for myocardium (red) regeneration. (B) When blocking blood flow or impairing cilia development, endocardial klf2 expression and Notch signaling activation are inhibited, which lead to failure of ventricle regeneration. (C) In klf2 mutants, the lack of endocardial klf2 gene expression affects the activation of Notch signaling, and the damaged ventricle cannot regenerate. A, atrium; AVC, atrioventricular canal; CL, cardiac lumen; EC, endocardium; KD, knockdown; MC, myocardium; V, ventricle.

form a complex to coordinately regulate Notch expression directly or indirectly, which requires further investigation in the future.

Primary cilia act as a link between hemodynamics and Notch signaling

Primary cilia on endothelium are involved in fluid flow sensation which can transduce shear stress signal into functional responses (Nauli et al., 2008). Liu et al. reported that primary cilia regulate hematopoietic stem and progenitor cell specification through Notch signaling in zebrafish (Liu et al., 2019). Samsa et al. demonstrated that primary cilia are present in the embryonic zebrafish endocardium at $30 \mathrm{hpf}$, and shear stress promotes the development of cardiac trabeculae by promoting the expression of notch $1 b$ through primary cilia (Samsa et al., 2015). So we speculate that primary cilia may participate in the mechanical shear force sensation and regulation of Notch signaling during cardiac regeneration at later stages. In this study we first confirmed the presence of cardiac cilia at 3 and 4 $\mathrm{dpf}$ in the endocardium of AVC region where Notch signaling is activated during heart regeneration. Primary cilia can also be detected in the ventricle, atrium and OFT, where they are not confined to endothelial cells but in the CMs and epicardial cells. The physiological functions these primary cilia possess are still unclear at this moment. Primary cilia formation is sensitive to flow speed and patterns (Egorova et al., 2011), and we did observe a highly dynamic pattern in the number of cardiac cilia. The cilia number in $4 \mathrm{dpf}$ heart is much less than that in $3 \mathrm{dpf}$ in physiological situation, but it increases after ventricle ablation and this increase can be blunted by inhibition of blood flow. Espinha et al. also demonstrated an increase in microtubules around primary cilia in response to oscillatory fluid flow stimulation (Espinha et al., 2014). Next we knocked down ift88, an intraflagellar transporter essential for the assembly and maintenance of cilia (Pazour et al., 2000), to 
inhibit cilia formation. Although ift88 morphant hearts still can contract and maintain low blood flow, the number of cilia is dramatically reduced. The expression of klf2 genes is downregulated and no Notch activation is observed in ift88 morphant hearts after ventricle injury, indicating the importance of cilia in this regenerative process. Recently Villalobos et al. also reported the presence of primary cilia in adult mouse, rat and human hearts where they play a pivotal role in pathological cardiac remodeling after injury (Villalobos et al., 2019).

How primary cilia regulate klf2 expression and affect Notch activation remains to be explored. Cilia are well known to participate in Hedgehog signaling during development (Rohatgi et al., 2007), they are also specialized calcium signal receptors that are highly sensitive to the low-frequency shear stress (Delling et al., 2013). There are a large number of ion channels on the primary cilia, like Piezo1/2, Polycystins 1/2, and other TRP channels (Orr et al., 2006). Piezo1 and piezo2 are important cation channels responsible for the mechanical activation in the somatosensory system (Coste et al., 2010). Polycystins $1 / 2$ can sense fluid flow and transduce signaling on primary cilia of mouse embryonic kidney epithelial cells (Nauli et al., 2003). Polycystins 2 (also known as Trpp2) and Trpv4 ion channels play a regulatory role between blood flow and klf2a gene expression during heart valve formation (Heckel et al., 2015) and trpv4 ${ }^{-/-}$mutants impede heart regeneration (Gálvez-Santisteban et al., 2019). However, whether these ion channels exist on the primary cilia of endocardial cells and how they participate in the regulation of heart regeneration warrant further investigation.

In summary, this study demonstrates primary cilia and flow responsive KIf2a/KIf2b factors as the mechanistic link between hemodynamic alteration and Notch signaling activation. Our findings reveal the pivotal role of mechanosensation pathway in regulating heart regeneration and provide novel insights and new directions for the treatment of ischemic heart diseases.

\section{METHODS}

\section{Zebrafish husbandry}

Zebrafish were raised and maintained under standard conditions. All experiments were performed according to institutional and national animal welfare guidelines. The zebrafish lines used in this study were as follows: $\operatorname{Tg}(t p 1: d 2 G F P)$ (Clark et al., 2012), Tg(vmhc: mCherry-NTR) (Zhang et al., 2013), Tg(Ubi:Arl13b-GFP) (Austin-Tse et al., 2013), $\operatorname{Tg}(f l k: G F P)$ (Jin et al., 2005), $T g$ (tcf21:nucGFP) (Mandal et al., 2017), ift20 $0^{-/-}$and ift172 $^{-1-}$ (courtesy of Dr Ying Cao). In all experiments, embryos and larvae over $24 \mathrm{hpf}$ were maintained in E3 water with 0.003\% PTU (1-phenyl-2-thiourea, Sigma, P7629) to prevent pigmentation.

\section{Generation of mutant zebrafish}

$k l f 2 a^{-1-}$ mutants, klf2 $b^{-1-}$ mutants and $k l f 2 a^{-1-} / k l f 2 b^{-1-}$ double mutants were generated using CRISPR/Cas9 technique (Chang et al., 2013). The Cas9 mRNA was generated by in vitro transcription from a linearized plasmid pT3TS-nls-zCas9-nls (CZRC, China) using a mMESSAGE mMACHINE kit (Ambion). sgRNA target sites were identified using web-based tool ZiFiT (http://zifit.partners.org). The sgRNAs were in vitro transcribed from the DNA template of PCRamplified products of the pMD19-T-gRNA vector (CZRC, China) with specific forward primers (klf2a: 5'-TGTAATACGACTCACTATAggtggaaggatgaactggacGTTTTAGAGCTAGAAATAGC-3' and klf2b: 5'TGTAATACGACTCACTATAggatgcaacccagcatgcagGTTTTAGAGCT AGAAATAGC-3'), and a universal reverse primer (5'-AAAAAAAGCACCGACTCGGTGCCACT-3'). A mixture of 300 pg Cas9 mRNA and 60 pg klf2a or klf2b sgRNA was injected into wildtype embryos at one-cell stage to obtain corresponding single mutants. Double mutants were generated by injecting Cas 9 mRNA and klf2 $b$ sgRNA into homozygous $k l f 2 a^{-/-}$mutant embryos. Positive founders were mated with wildtype fish to obtain F1 generation. The F1 heterozygous zebrafish with identical frameshift mutations were intercrossed to generate $\mathrm{F} 2$ homozygous mutants.

\section{Chemical treatment}

Tg(vmhc:mCherry-NTR) larvae at $72 \mathrm{hpf}$ were treated with $6 \mathrm{mmol} / \mathrm{L}$ MTZ (Metronidazole, Sigma) in E3 water for $4 \mathrm{~h}$ as previously described (Zhang et al., 2013). As controls, age-matched Tg(vmhc: $m$ Cherry-NTR) siblings were incubated in $0.2 \%$ DMSO (dimethyl sulfoxide, Fisher Scientific) in E3 water for the same period. To stop blood flow, control or ablated Tg(vmhc:mCherry-NTR) larvae were treated with $1.8 \mathrm{mmol} / \mathrm{L}$ Tricaine (3-aminobenzoic acid ethyl ester, Sigma) or $10 \mathrm{mmol} / \mathrm{L}$ BDM (2,3-Butanedione monoxime, Sigma) in E3 water from $15 \mathrm{hpt}$ for $9 \mathrm{~h}$ at $28^{\circ} \mathrm{C}$. Treated larvae were washed three times with fresh E3 water at the end of ablation, Tricaine or BDM treatment and then allowed to continue to grow in fresh E3 water.

\section{In situ hybridization}

Whole mount in situ hybridization was performed as previously described (Zhang et al., 2013), using the following probes: notch1b, $k l f 2 a$, hand2, $n k x 2.5$. The primers used for $k l f 2 b$ probe synthesis were: forward primer $5^{\prime}$-GAATTCCGCACACAATTGGTCTAGGA-3' and reverse primer 5'-GGTAATACGACTCACTATAGGTACGTACATCGTTGTGCATTTTCCAC-3'.

\section{Immunofluorescence}

Immunofluorescence staining on dissected larval hearts or whole mount larvae was performed as previously described (Zhang et al., 2013). The primary antibodies used in this study include: anti-GFP (chicken; Abcam, ab13970), anti-acetylated alpha tubulin (mouse; Abcam, ab24610), anti-Arl13b (rabbit; Proteintech, 17711-1-AP). The secondary antibodies used in this study include: Alexa Fluor 488 goat anti-mouse lgG, Alexa Fluor 488 goat anti-rabbit lgG, Alexa Fluor 555 goat anti-mouse lgG, Alexa Fluor 555 goat anti-rabbit lgG and Alexa Fluor 488 goat anti-chicken IgG from Invitrogen. Fluorescent images were obtained using a Leica SP8 or Zeiss LSM710 or Zeiss LSM880 confocal microscope. 


\section{Morpholino injection}

Morpholino injections were performed as previously described (Samsa et al., 2015). The morpholinos against ift88 (5'-CTGGGACAAGATGCACATTCTCCAT-3') was purchased from GeneTools. 5 ng ift88 MO was injected into embryos at one-cell stage. Embryos and larvae at specific stages were used for relevant experiments.

\section{Quantitative real-time PCR}

qPCR analysis for global expression of $k l f 2 a$ and $k l f 2 b$ was performed on cDNA obtained from $4 \mathrm{dpf}$ wildtype, $\mathrm{klf}^{2} \mathrm{a}^{-/-}$mutants, $k l f 2 b^{-1-}$ mutants and $k l f 2^{-1-}$ double mutants. Total RNA was extracted by homogenizing 30 embryos in TRIzol reagent (Invitrogen) with a TGrinder pestle (Tiangen). $2 \mu \mathrm{g}$ total RNA was used for cDNA synthesis with a ReverTra Ace qPCR RT Kit (TOYOBO). All realtime PCR reactions were performed in quadruplicate with Pow$\operatorname{erUp}^{\mathrm{TM}}$ SYBR $\circledast$ Green Master Mix (Thermo Fisher) and three independent biological repeats were performed. Gene expression values were normalized using $\beta$-actin as internal control. For relative quantification analysis, statistical significance was determined by ANOVA analysis. The primers used in this study were as follows: klf2a forward primer 5'-GCTGGGAGAACAGGTGGAAGG-3', reverse primer 5'-GCCATGCCGAGTCCGAGATT-3'; klf2b forward primer 5'-ATGCAGCGGGCTCTTCTCAC-3', reverse primer 5'TTTTCACCGGTGTGAGTGCG-3'.

\section{Quantification and statistical analysis}

The regeneration ratio was calculated as the number of recovered larvae over the number of total injured larvae. Values were presented as mean \pm s.e.m. Statistical significance was defined as a threshold of $P<0.05$ determined by Student's $t$-test between two groups, ANOVA analysis between more than two groups or Binomial test in quantification of the percentage of recovered hearts.

\section{ACKNOWLEDGEMENTS}

We thank Haitao Zhou and Lifeng Li for fish care, Kaa Seng Lai, Yabo Fang and Wenyan Li for technical support and other lab members for in depth discussion. We thank $\mathrm{Dr}$ Tao Zhong for providing reagents. This study was supported by National Key R\&D Program of China grant 2018YFA0801004 and NSFC grant 31571492 to R.Z.

\section{AUTHOR CONTRIBUTIONS}

X.L and R.Z conceived and designed the project. X.L, Q.L, Y.P, F.G, $X . S$, and H.Z conducted the experiments. Y.C provided critical reagents. X.L and R.Z wrote and revised the manuscript. All authors reviewed the manuscript.

\section{ABBREVIATIONS}

AVC, atrioventricular canal; BDM, 2,3-butanedione monoxime; CMs, cardiomyocytes; dpf, days post fertilization; dpt, days post treatment; DMSO, dimethyl sulfoxide; hpf, hours post fertilization; hpt, hours post treatment; MO, morpholino; MTZ, metronidazole; OFT, outflow tract; PIV, particle image velocimetry; PTU, phenylthiourea; WISH, whole-mount in situ hybridization.

\section{COMPLIANCE WITH ETHICAL GUIDELINES}

Xueyu Li, Qiang Lu, Yuanyuan Peng, Fang Geng, Xuelian Shao, Huili Zhou, Ying Cao, and Ruilin Zhang declare that they have no conflict of interest. All institutional and national guidelines for the care and use of laboratory animals were followed.

\section{OPEN ACCESS}

This article is licensed under a Creative Commons Attribution 4.0 International License, which permits use, sharing, adaptation, distribution and reproduction in any medium or format, as long as you give appropriate credit to the original author(s) and the source, provide a link to the Creative Commons licence, and indicate if changes were made. The images or other third party material in this article are included in the article's Creative Commons licence, unless indicated otherwise in a credit line to the material. If material is not included in the article's Creative Commons licence and your intended use is not permitted by statutory regulation or exceeds the permitted use, you will need to obtain permission directly from the copyright holder. To view a copy of this licence, visit http:// creativecommons.org/licenses/by/4.0/.

\section{REFERENCES}

Atkins GB, Jain MK (2007) Role of Kruppel-like transcription factors in endothelial biology. Circ Res 100(12):1686-1695

Austin-Tse C, Halbritter J, Zariwala MA, Gilberti RM, Gee HY, Hellman N, Pathak N, Liu Y, Panizzi JR, Patel-King RS et al (2013) Zebrafish ciliopathy screen plus human mutational analysis identifies C21orf59 and CCDC65 defects as causing primary ciliary dyskinesia. Am J Hum Genet 93(4):672-686

Baratchi S, Khoshmanesh K, Woodman OL, Potocnik S, Peter K, Mclntyre P (2017) Molecular sensors of blood flow in endothelial cells. Trends Mol Med 23(9):850-868

Chang N, Sun C, Gao L, Zhu D, Xu X, Zhu X, Xiong J-W, Xi JJ (2013) Genome editing with RNA-guided Cas9 nuclease in zebrafish embryos. Cell Res 23(4):465-472

Clark BS, Cui S, Miesfeld JB, Klezovitch O, Vasioukhin V, Link BA (2012) Loss of Llgl1 in retinal neuroepithelia reveals links between apical domain size, Notch activity neurogenesis. Development 139(9):1599-1610

Coste B, Mathur J, Schmidt M, Earley TJ, Ranade S, Petrus MJ, Dubin AE, Patapoutian A (2010) Piezo1 and Piezo2 are essential components of distinct mechanically activated cation channels. Science 330(6000):55-60

Dekker RJ, van Soest S, Fontijn RD, Salamanca S, de Groot PG, VanBavel E, Pannekoek H, Horrevoets AJ (2002) Prolonged fluid shear stress induces a distinct set of endothelial cell genes, most specifically lung Krüppel-like factor (KLF2). Blood 100(5):16891698 
Delling M, DeCaen PG, Doerner JF, Febvay S, Clapham DE (2013) Primary cilia are specialized calcium signalling organelles. Nature 504(7479):311

Egorova AD, Khedoe PP, Goumans MJ, Yoder BK, Nauli SM, ten Dijke P, Poelmann RE, Hierck BP (2011) Lack of primary cilia primes shear-induced endothelial-to-mesenchymal transition. Circ Res 108(9):1093-1101

Espinha LC, Hoey DA, Fernandes PR, Rodrigues HC, Jacobs CR (2014) Oscillatory fluid flow influences primary cilia and microtubule mechanics. Cytoskeleton 71(7):435-445

Felician G, Collesi C, Lusic M, Martinelli V, Ferro MD, Zentilin L, Zacchigna S, Giacca M (2014) Epigenetic modification at Notch responsive promoters blunts efficacy of inducing Notch pathway reactivation after myocardial infarction. Circ Res 115(7):636-641

Goddard LM, Duchemin AL, Ramalingan H, Wu B, Chen M, Bamezai S, Yang J, Li L, Morley MP, Wang T et al (2017) Hemodynamic forces sculpt developing heart valves through a KLF2-WNT9B paracrine signaling axis. Dev Cell 43(3):274-289

Gálvez-Santisteban M, Chen D, Zhang R, Serrano R, Nguyen C, Zhao L, Nerb L, Masutani EM, Vermot J, Burns CG et al (2019) Hemodynamic-mediated endocardial signaling controls in vivo myocardial reprogramming. eLife 8:e44816

Heckel E, Boselli F, Roth S, Krudewig A, Belting H-G, Charvin G, Vermot $J$ (2015) Oscillatory flow modulates mechanosensitive klf2a expression through trpv4 and trpp2 during heart valve development. Curr Biol 25(10):1354-1361

Jin S-W, Beis D, Mitchell T, Chen J-N, Stainier DY (2005) Cellular and molecular analyses of vascular tube and lumen formation in zebrafish. Development 132(23):5199-5209

Jopling C, Sleep E, Raya M, Marti M, Raya A, Belmonte JCI (2010) Zebrafish heart regeneration occurs by cardiomyocyte dedifferentiation and proliferation. Nature 464(7288):606

Kikuchi K, Holdway JE, Werdich AA, Anderson RM, Fang Y, Egnaczyk GF, Evans T, Macrae CA, Stainier DYR, Poss KD (2010) Primary contribution to zebrafish heart regeneration by gata4(+) cardiomyocytes. Nature 464(7288):601-605

Lee JS, Yu Q, Shin JT, Sebzda E, Bertozzi C, Chen M, Mericko P, Stadtfeld M, Zhou D, Cheng $L$ et al (2006) Klf2 is an essential regulator of vascular hemodynamic forces in vivo. Dev Cell 11 (6):845-857

Liu Z, Tu H, Kang Y, Xue Y, Ma D, Zhao C, Li H, Wang L, Liu F (2019) Primary cilia regulate hematopoietic stem and progenitor cell specification through Notch signaling in zebrafish. Nat Commun 10(1):1839

Mandal A, Holowiecki A, Song YC, Waxman JS (2017) Wnt signaling balances specification of the cardiac and pharyngeal muscle fields. Mech Dev 143:32-41

Munch J, Grivas D, Gonzalez-Rajal A, Torregrosa-Carrion R, de la Pompa JL (2017) Notch signalling restricts inflammation and serpine1 expression in the dynamic endocardium of the regenerating zebrafish heart. Development 144(8):1425-1440

Nauli SM, Alenghat FJ, Luo Y, Williams E, Vassilev P, Li X, Elia AEH, Lu W, Brown EM, Quinn SJ et al (2003) Polycystins 1 and 2 mediate mechanosensation in the primary cilium of kidney cells. Nat Genet 33(2):129
Nauli SM, Kawanabe Y, Kaminski JJ, Pearce WJ, Ingber DE, Zhou J (2008) Endothelial cilia are fluid shear sensors that regulate calcium signaling and nitric oxide production through polycystin1. Circulation 117(9):1161-1171

Nemir M, Metrich M, Plaisance I, Lepore M, Cruchet S, Berthonneche C, Sarre A, Radtke F, Pedrazzini T (2014) The Notch pathway controls fibrotic and regenerative repair in the adult heart. Eur Heart J 35(32):2174-2185

Nixon AM, Gunel M, Sumpio BE (2010) The critical role of hemodynamics in the development of cerebral vascular disease: a review. J Neurosurg 112(6):1240-1253

Oates AC, Pratt SJ, Vail B, Yan Y, Ho RK, Johnson SL, Postlethwait $\mathrm{JH}$, Zon LI (2001) The zebrafish klf gene family. Blood 98 (6):1792-1801

Orr AW, Helmke BP, Blackman BR, Schwartz MA (2006) Mechanisms of mechanotransduction. Dev Cell 10(1):11-20

Pazour GJ, Dickert BL, Vucica Y, Seeley ES, Rosenbaum JL, Witman GB, Cole DG (2000) Chlamydomonas IFT88 and its mouse homologue, polycystic kidney disease gene $\operatorname{tg} 737$, are required for assembly of cilia and flagella. J Cell Biol 151(3):709718

Poss KD, Wilson LG, Keating MT (2002) Heart regeneration in zebrafish. Science 298(5601):2188-2190

Rasouli SJ, El-Brolosy M, Tsedeke AT, Bensimon-Brito A, Ghanbari P, Maischein HM, Kuenne C, Stainier DY (2018) The flow responsive transcription factor KIf2 is required for myocardial wall integrity by modulating Fgf signaling. eLife 7:e38889

Raya A, Koth CM, Büscher D, Kawakami Y, Itoh T, Raya RM, Sternik G, Tsai H-J, Rodríguez-Esteban C, Izpisúa-Belmonte JC (2003) Activation of Notch signaling pathway precedes heart regeneration in zebrafish. Proc Natl Acad Sci USA 100(Suppl 1):1188911895

Rohatgi R, Milenkovic L, Scott MP (2007) Patched1 regulates hedgehog signaling at the primary cilium. Science 317 (5836):372-376

Samsa LA, Givens C, Tzima E, Stainier DY, Qian L, Liu J (2015) Cardiac contraction activates endocardial Notch signaling to modulate chamber maturation in zebrafish. Development 142 (23):4080-4091

Steed E, Faggianelli N, Roth S, Ramspacher C, Concordet J-P, Vermot J (2016) klf2a couples mechanotransduction and zebrafish valve morphogenesis through fibronectin synthesis. Nat Commun 7:11646

Thygesen K, Alpert JS, White HD (2007) Universal definition of myocardial infarction. J Am Coll Cardiol 50(22):2173-2195

Vermot J, Forouhar AS, Liebling M, Wu D, Plummer D, Gharib M, Fraser SE (2009) Reversing blood flows act through klf2a to ensure normal valvulogenesis in the developing heart. PLoS Biol 7(11):e1000246

Villalobos E, Criollo A, Schiattarella GG, Altamirano F, French KM, May HI, Jiang N, Nguyen NUN, Romero D, Roa JC et al (2019) Fibroblast primary cilia are required for cardiac fibrosis. Circulation 139:2342-2357

Zhang R, Han P, Yang H, Ouyang K, Lee D, Lin YF, Ocorr K, Kang G, Chen J, Stainier DY et al (2013) In vivo cardiac reprogramming 
contributes to zebrafish heart regeneration. Nature 498 (7455):497-501

Zhao L, Ben-Yair R, Burns CE, Burns CG (2019) Endocardial Notch signaling promotes cardiomyocyte proliferation in the regenerating zebrafish heart through Wnt pathway antagonism. Cell Rep 26(3):546-554
Zhao L, Borikova AL, Ben-Yair R, Guner-Ataman B, MacRae CA, Lee RT, Burns CG, Burns CE (2014) Notch signaling regulates cardiomyocyte proliferation during zebrafish heart regeneration. Proc Natl Acad Sci USA 111(4):1403-1408 